is naturaleza 


\section{DOSSIER CRÍTICO SOBRE EL LIBRO:}

\section{LOS SÓTANOS DEL UNIVERSO}

Volumen monográfico de

\section{NATURALEZA Y LIBERTAD}

Revista de estudios interdisciplinares

Número 3

Málaga, 2014

Esta revista es accesible on-line en el siguiente portal: http://grupo.us.es/naturalezayl 


\section{Naturaleza y Libertad}

Revista de estudios interdisciplinares

Número 3, 2014

ISSN: 2254-96682014

Directores: Juan Arana, Universidad de Sevilla; Juan José Padial, Universidad de Málaga; Francisco Rodríguez Valls, Universidad de Sevilla.

Secretaria: Avelina Cecilia Lafuente, Universidad de Sevilla.

Consejo de Redacción: José Luis González Quirós, Universidad Juan Carlos I, Madrid; Francisco Soler, Universität Dortmund / Universidad de Sevilla; Pedro Jesús Teruel. Universidad CEU Cardenal Herrera; Héctor Velázquez, Universidad Panamericana, México.

Adjunto a la redacción: Miguel Palomo, Universidad de Sevilla

Consejo Editorial: Mariano Álvarez, Real Academia de Ciencia Morales y Políticas; Allan Franklin, University of Colorado; Michael Heller, Universidad Pontificia de Cracovia; Manfred Stöcker, Universität Bremen; William Stoeger, University of Arizona.

Consejo Asesor: Rafael Andrés Alemañ Berenguer. Universidad de Alicante; Juan Ramón Álvarez, Universidad de León; Luciano Espinosa, Universidad de Salamanca; Miguel Espinoza, Université de Strasbourg; Juan A. García González, Universidad de Málaga; José Manuel Giménez Amaya, Universidad de Navarra; Karim Gherab Martín, Urbana University, Illinois; Martín López Corredoira, Instituto de Astrofísica de Canarias; Alfredo Marcos, Universidad de Valladolid; Javier Monserrat, Universidad Autónoma de Madrid; Leopoldo Prieto, Colegio Mayor San Pablo, Madrid; Ana Rioja, Universidad Complutense, Madrid. Madrid; José Luis González Recio, Universidad Complutense, Madrid; Javier Serrano, TEC Monterrey (México); Hugo Viciana, Université Paris I; Claudia Vanney, Universidad Austral, Buenos Aires; José Domingo Vilaplana, Huelva.

\section{Redacción y Secretaría:}

Naturaleza y Libertad. Revista de estudios interdisciplinares. Departamento de Filosofía y Lógica. Calle Camilo José Cela s.n. E-41018 Sevilla.

Depósito Legal: MA2112-2012

용 954.55.77.57 Fax: 954.55.16.78. E-mail: jarana@us.es 
Naturaleza y Libertad. Revista de estudios interdisciplinares. Número 3, 2014. ISSN: 2254-9668

\section{ÍNDICE}

PRESENTACIÓN.

DOSSIER CRÍTICO SOBRE EL LIBRO LOS SÓTANOS DEL UNIVERSO

Juan Arana (U. de Sevilla), El problema de la determinación y el diálogo interdisciplinar......11

Miguel Acosta (U. CEU San Pablo, Madrid), La epistemología del riesgo y la

relación ciencia-filosofía. . .25

Carlos del Ama (Madrid), Reflexiones con ocasión del libro Los sótanos del universo ...........57

Luciano Espinosa (U. de Salamanca), De los sótanos al ático del universo ............................93

Gonzalo Génova (U. Carlos III, Madrid), Turing y el rostro del universo ..........................101

Julio A. Gonzalo (U. Autónoma de Madrid), De los sótanos a los confines del universo ....111

Juan Meléndez Sánchez (U. Carlos III, Madrid), De riesgos y metáforas

Andrés Muñoz Machado (Universidad Politécnica de Madrid), Las matemáticas en

Los sótanos del universo

Javier Sánchez Cañizares (U. de Navarra), Sótanos del universo, espejos del alma

Francisco José Soler Gil (U. de Sevilla), Una filosofía de la naturaleza contracorriente y antisistema.

Ignacio Sols (U. Complutense, Madrid), Recuperando la causa formal sin abandonar la causa eficiente. .151

Juan Arana (U. de Sevilla), Los subsótanos del universo. Materiales para un debate. 167 
Naturaleza y Libertad. Revista de estudios interdisciplinares. Número 3, 2014. ISSN: 2254-9668

\title{
EL PROBLEMA DE LA DETERMINACIÓN Y EL DIÁLOGO INTERDISCIPLINAR ${ }^{1}$ \\ Juan Arana \\ Universidad de Sevilla
}

\begin{abstract}
Resumen: El artículo los objetivos y naturaleza del libro Los sótanos del universo, y destaca los aspectos que pueden ser de mayor interés para filósofos (muy en particular, los que se adscriben al aristotelismo), científicos, teólogos y público en general.

Palabras clave: Filosofía de la naturaleza, interdisciplinariedad, epistemología del riesgo.

Abstract: This paper presents the objectives and nature of the book Los Sótanos del Universo and emphasises the aspects that could be center of interest for philosophers (and in particular those who view themselves as aristotelians), scientists, theologians and people in general.
\end{abstract}

Keywords: Philosophy of Nature, Interdisciplinarity, Epistemology of Risk.

Recibido: 20 de octubre de 2012. Aprobado: 12 de diciembre de 2013.

El año 1977 comencé a explicar la asignatura de "filosofía de la naturaleza” en la Universidad de Sevilla. Llevo pues la friolera de 35 años enseñándola. Es una especie de "cenicienta" dentro de los estudios de filosofía y la mayoría de los colegas la considera una supervivencia anacrónica de tiempos pasados. Algunos amigos - para hacerme quedar bien - dicen que lo que hago en realidad es "filosofía de la ciencia", disciplina que suena mucho más actual. Pero no: lo mío es la filosofía de la naturaleza y no tengo

1 J. Arana, Los sótanos del Universo. La determinación natural y sus mecanismo ocultos, Madrid, Editorial Biblioteca Nueva, Col. Manuales Universidad, 2012. ISBN: 978-84-9940-479-0. 400 pp. 
ninguna intención de cambiarla por otra. Cualquiera podrá añadir además que ya se me ha pasado el arroz: dentro de tres años podré jubilarme si quiero (con crisis económica y todo) y dentro de ocho me jubilarán aunque no quiera.

Para reivindicarme diré que en su momento elegí esta materia y, a pesar de los años transcurridos, sigo tercamente aficionado a ella. Los únicos manuales que había en el mercado cuando empecé eran "tomistas", o bien se trataba de meras historias de la ciencia camufladas. Pronto nació en mí la inquietud de escribir una exposición más de mi gusto, y en 1996-2000 la publiqué en dos volúmenes titulados Claves del conocimiento del mundo. En 2001 salió una versión mejorada, Materia, universo, vida, que mis alumnos han padecido hasta hoy. En adelante sufrirán con el nuevo libro, ya que por sus dimensiones sólo resultaba publicable si se adornaba con la pretensión de constituir, en efecto, un "manual”. Me considero una persona mínimamente honrada y desde luego en cierto modo lo es. Pero al mismo tiempo es otra cosa: nada menos que el libro que siempre pensé escribir "cuando fuera mayor". Ya corría el riesgo de hacerme demasiado mayor, así que hace un par de años me puse las pilas y ahí lo tienen. En los momentos de mayor optimismo soñé hacer algo mucho más importante; en las horas bajas temí quedarme incluso por debajo de éste. Alimento la ilusión de que el resultado final haya alcanzado un discreto término medio y considero que, además de informar a mis estudiantes de las cuestiones cruciales relativas al universo, contiene un resumen de todo lo que he aprendido después de tantos años. Hasta puede que interese a algunos colegas y a esos raros especímenes de la raza humana que practican la obra de misericordia más admirable de todas, aunque no 
figure en la lista oficial: leer sin más ni más, porque sí, los libros que escribe el prójimo.

A mis alumnos, ocioso es decirlo, ya los tengo atrapados con la amenaza implícita de suspenderlos si no leen el libro de punta a cabo. Pero ¿y los demás? Se me da muy mal el marketing. Doy por seguro que, si tengo que pasar una temporada en el purgatorio, se me obligará allí a vender coches, enciclopedias o seguros. Entenderán por tanto mi repugnancia a la idea de promocionar la venta de mi libro. Pero me siento en deuda con el editor que ha creído en él, así que voy a efectuar una simbólica campaña publicitaria en forma de un manifiesto dirigido a cada uno de los grupos sociales que pueden ser remotamente susceptibles de claudicar ante los hechizos de la criatura.

\section{1. ¿QUÉ INTERÉS PUEDE TENER EL LIBRO PARA UN FILÓSOFO?}

El libro intenta avanzar hacia una "superación de Kant" todavía pendiente. El filósofo alemán formuló una objeción de principio contra cualquier indagación teórica que involucrara los conceptos de "universo", “yo" o "Dios", por tratarse de conceptos de imposible validación empírica. Como máximo les reconocía un desvaído "uso regulativo", insuficiente para constituir disciplinas con pretensión de verdad objetiva. Desde finales del siglo XVIII han sido muy pocos los autores que se han atenido a las enseñanzas de Kant en la mayor parte de las cuestiones. Sin embargo, la deslegitimación de la filosofía de la naturaleza, la antropología filosófica y la teología natural ha sido un presupuesto compartido por la mayor parte del colectivo filosófico. Ha reinado en este punto un consenso práctico, no sólo dentro de las escuelas que se mueven en el ámbito de lo que podríamos llamar "filosofía trascendental", 
sino también entre las que siguen una orientación más empirista e incluso las que reivindican la metafísica como ciencia teórica sustantiva. Así pues, se ha producido una "kantianizacion" de la filosofía reconocible en cuatro rasgos principales:

a) La tendencia a asignar a la ciencia positiva la competencia exclusiva sobre todos los juicios basados en la experiencia.

b) El criterio de que sólo la certeza apodíctica es aceptable para el filósofo.

c) El rechazo de lo que a partir de Wolff se ha dado en llamar "ontologías regionales" (esto es, filosofía de la naturaleza, antropología filosófica y teología natural).

d) La transformación de la filosofía en una actividad de tipo subjetivopráctico-introspectivo, o bien en una teoría vacía de contenido ontológico y vuelta hacia lo epistemológico y/o lingüístico.

En Los sótanos del universo me aparto de la orientación dominante y tacho de obsoletos estos planteamientos. Defiendo entre otras las siguientes tesis:

a) No existen juicios sintéticos a priori en parte alguna de las ciencias que se ocupan de la realidad (empezando por la propia física).

b) En las ciencias naturales no hay un solo enunciado que posea certeza apodíctica, lo cual no les ha impedido alcanzar el estatuto de validez cognitiva objetiva.

c) Por consiguiente, tampoco hay que esperar encontrar juicios sintéticos a priori en ontología, ni debe exigirse certezas apodícticas en todo lo que dentro de la filosofía se refiere al conocimiento de la realidad.

d) El rigor de la llamada —al menos desde Descartes hasta Husserl"ciencia estricta" puede tal vez encontrarse en las ciencias formales. En cambio, es imperativo desarrollar una epistemología del riesgo para uso tanto 
de las ciencias positivas como de las partes de la filosofía que tengan algún contenido de verdad (entendiendo ésta como adecuación a la realidad extramental).

e) No existen fronteras netamente definidas entre las ciencias positivas y la filosofía, en el sentido de que ambas se apoyan en las aptitudes naturales del hombre y en la experiencia tanto externa como interna. Las diferencias entre ambas disciplinas son menos formales que materiales. Dependen sobre todo de contingencias históricas: llamamos saberes positivos a las disciplinas que han precisado su objeto de estudio mediante la definición de conceptos y la elaboración de métodos de alcance limitado. Por su lado, compete a la filosofía efectuar elaboraciones teóricas abiertas, con restricciones conceptuales y metodológicas menos marcadas y más provisionales.

Dentro de este amplio programa de renovación filosófica, el libro quiere ubicarse en una filosofía de la naturaleza a la altura de los tiempos. Está centrado en el problema de la determinación de los procesos físicos y en el examen y crítica de las categorías usadas tanto por la ciencia como por la filosofía a este propósito: causa, ley, azar, complejidad, reducción, emergencia, determinismo y contingentismo. El diálogo entre lo científico y lo filosófico es permanente, así como la interacción entre la instancia histórica y la teórica. Se efectúa una breve incursión en la epistemología del riesgo, disciplina en buena parte pendiente de consolidación teórica, a fin de establecer los presupuestos mínimos de una investigación cuyo centro de gravedad es la ontología del ente físico. 


\section{2. ¿QUÉ INTERÉS PUEDE TENER EL LIBRO PARA UN CIENTÍFICO?}

Desde mediados del siglo XVIII los filósofos dejaron en manos de los científicos, gremio entonces apenas constituido y consolidado, la exclusiva del conocimiento positivo de la realidad. No sólo eso sino que, tras algunos grandiosos pero frustrados esfuerzos por conocer esa misma realidad de un modo especulativo (¿?), en la práctica les dejaron libre todo el campo, para consagrarse a abstrusas incursiones en el mundo de la reflexión, la crítica, la sospecha y la reforma ética o social.

Aunque por oficio y hábito el científico tiende a contenerse a la hora de generalizar, la tentación de explorar el panorama que se abría más allá de sus teorías y experimentos era demasiado grande, de manera que desde principios del siglo XIX empezaron a proliferar "cosmovisiones científicas", esto es, filosofías elaboradas como extrapolación o a título de complemento de las teorías científicas de mayor enjundia y alcance.

A propósito de este hecho se han barajado dos posturas:

a) La primera defiende la continuidad pura y simple de los asuntos investigados con los conceptos y métodos de las ciencias positivas y las cuestiones últimas relativas a la estructura, origen y destino de la realidad que percibimos. Desemboca casi siempre en concepciones naturalistas y materialistas.

b) La segunda pretende que el científico debe inhibirse ante cuestiones que desbordan la operatividad de sus instrumentos conceptuales y empíricos, de lo que se deriva una discontinuidad muy neta entre la óptica científica y la filosófica, sobre la que se pueden mantener las más diversas posiciones, 
dejando siempre a salvo que nada tienen que ver con lo que el científico averigua.

En la evolución de la ciencia en los últimos dos siglos se han producido acontecimientos que parecen avalar tanto una postura como otra. Por un lado, el nacimiento y desarrollo de la cosmología científica a partir de Einstein refuerza el criterio de que la noción de "universo" no es una idea trascendental de la razón como pretenden muchos filósofos, sino un concepto perfectamente homologable con los que emplea la ciencia. Por otro lado, el desarrollo de la mecánica cuántica ha impuesto límites objetivos al uso de los conceptos físicos fundamentales, lo que impide extrapolarlos más allá de un determinado límite.

Estos y muchos otros indicios permiten contemplar una tercera vía, todavía poco explorada: consiste en defender que hay continuidad entre la perspectiva científica y la filosófica, sin que por ello sea obligado asumir un compromiso teórico naturalista. El naturalismo es la actitud propia del científico cuando se mueve dentro de su propio campo, pero cuando asume el naturalismo como opción filosófica pierde la oportunidad de distanciarse de sí mismo y ser autocrítico. Si por otro lado su renuncia a la opción preferente por el naturalismo no le fuerza a perder o considerar inútiles los frutos de su trabajo científico cuando inicia su andadura filosófica, la situación se vuelve realmente interesante.

En los Sótanos del universo he tratado de llevar a cabo el siguiente experimento intelectual: no renunciar a nada de lo que la ciencia ofrece, pero tampoco aceptar la hipoteca del prejuicio naturalista. La inexistencia de un telón de acero para separar lo científico de lo filosófico no implica que cuando avanzamos más allá de los límites de la ciencia necesariamente ten- 
gamos que encontrar más de lo mismo. El paisaje va cambiando poco a poco, aunque no de un modo abrupto, y podemos descubrir grandes y gratas novedades si resistimos al desfallecimiento y nos dejamos sorprender...

\section{3. ¿QUÉ INTERÉS PUEDE TENER EL LIBRO PARA UN TEÓLOGO?}

En la introducción advierto que, aunque el concepto de "Dios" se usa con frecuencia a lo largo de Los sótanos del universo, no pretendo resolver ni dar por resuelta la pregunta por la existencia y atributos de la divinidad. Y ello a pesar de que personalmente asumo la tesis de que tras el universo cabe inferir razonablemente la presencia de un Dios personal. $\mathrm{Y}$ es que no he escrito un tratado de metafísica general ni tampoco de teología natural, sino un libro de filosofía de la naturaleza que pretende devolver a esta disciplina toda la carga teórica que tenía antaño.

He de advertir, no obstante, que en modo alguno intento retornar a un estadio "prekantiano" en la historia de la disciplina, ya que defiendo que la irrupción de la ciencia moderna ha supuesto un enriquecimiento sustancial en nuestro conocimiento del mundo físico, aunque las filosofías modernas y contemporáneas no hayan sabido asumirlo, debido a que han optado mayoritariamente por la ruptura de la unidad del conocimiento.

El libro intenta recuperar de algún modo ese paradigma perdido y restablecer los lazos orgánicos que debiera haber entre lo científico y lo filosófico. Defiende por consiguiente que los descubrimientos de la ciencia de Copérnico para acá sí tienen relevancia filosófica y que compete al filósofo valorarla. Conviene unir las enseñanzas provenientes de este campo a las aportaciones de los metafísicos y de los estudiosos del hombre, a fin de obte- 
ner una síntesis teórica que haga justicia al esfuerzo de búsqueda de la verdad realizado por todas las disciplinas y épocas.

Como el lector habrá ya adivinado, los objetivos directos que persigo son mucho más modestos. Intento imprimir un "giro ontológico" al estudio filosófico del hecho científico frente al "enfoque epistemológico" hasta ahora en circulación: por eso el libro no se inscribe en la filosofía de la ciencia, sino en la filosofía de la naturaleza, cuyas implicaciones antropológicas y teológicas son más claras. Con respecto a la teología se sitúa en el terreno de los preambula fidei y de sus fundamentos, porque el conocimiento en profundidad de la naturaleza es un elemento importante para conocer el hombre que la habita y para indagar el principio o principios de los que resulta.

Los argumentos cosmológicos fueron en otro tiempo parte importante de la teología natural. Después de un largo paréntesis están adquiriendo de nuevo candente actualidad. Con todo, son numerosísimos los obstáculos que hay que superar para evitar que la discusión naufrague en los prejuicios e ignorancias de que adolecen los interlocutores en este debate. El desinterés hacia el hecho científico por parte de la tradición teológica y metafísica en los últimos siglos ha motivado que el naturalismo y el materialismo hayan prosperado como la única cosmovisión que está en armonía con la física y la biología modernas.

Muchos defensores de la teología han querido deslegitimizar estas corrientes por considerarlas extrapolaciones ilegítimas de un saber de alcance muy restringido, pero su estrategia ha producido resultados desastrosos. Lo que proponían era, en definitiva una teoría de la doble verdad que, ni sirvió para nada bueno a finales de la Edad Media, ni tampoco lo está haciendo en estos momentos. El decaimiento de la teología fundamental tiene mucho que 
ver con esta pérdida de los horizontes filosóficos de la ciencia, así como de los horizontes empíricos de la filosofía. Hay que empezar desde muy abajo para restaurar una situación que no debió nunca deteriorarse como la pasividad de unos y el sectarismo de otros han provocado.

\section{4. ¿QUÉ INTERÉS PUEDE TENER EL LIBRO PARA UN NO ESPECIALISTA?}

Vivimos un mundo y una cultura llenos de escisiones. La más conocida opone las ciencias a las humanidades, y aunque todos lamentan el fenómeno casi nadie defiende que tenga remedio ni hace nada para propiciarlo. La excusa que damos para justificar nuestra pasividad es que los conocimientos han proliferado tanto que de ninguna manera caben en una sola cabeza. Pero este argumento, digamos, "cuantitativo", no debe ser muy convincente, puesto que son muchos los que se dedican a levantar muros entre las disciplinas, parecidos al que antaño dividía Berlín y todavía hoy separan territorios vecinos mutuamente hostiles.

No me considero un especialista sólido en ninguna de las grandes ramas del saber, ni tampoco un periodista o un divulgador experto en comunicar al lego lo que averigua el docto. Sólo he procurado establecer prioridades y jerarquías, localizar los hallazgos verdaderamente importantes, averiguar las cosas que nadie debería ignorar, rescatarlas de los oscuros añadidos que impiden su difusión y buscar su encaje con otras verdades igualmente importantes, pero actualmente desperdigadas en el caótico mundo del conocimiento. Pretendo derribar muros lingüísticos y conceptuales, lograr en definitiva que los árboles no nos impidan ver el bosque. 
La filosofía en sus mejores tiempos no fue una profesión, sino una afición: el gusto por la verdad. Los sótanos del universo es un libro que intenta recuperar ese estilo lúdico y a la vez intensamente comprometido a la hora de buscar respuestas a las preguntas que a todos importan. Por eso está lleno de discusiones con sabios y pensadores de ayer o de hoy, hombres que admiro y respeto pero con los que con mucha frecuencia no estoy de acuerdo. Discutir por discutir es estéril y descorazonador, pero de la discusión sale la luz cuando los contendientes no tratan de que sus opciones teóricas prevalezcan, sino que pretenden hallar cuál es la mejor opción para asumirla en tanto no aparezca otra mejor.

Si fuésemos capaces de echar abajo la montaña de dogmatismos y lugares comunes en que individual y colectivamente nos hemos ido encerrando tras dos milenios y medio de maravillosas aventuras intelectuales, seguramente tendríamos la fortuna de descubrir que, además de saber muchísimas cosas de importancia subalterna, también tenemos elementos de juicio para hacer apuestas razonables en las que son verdaderamente cruciales. Aunque el libro empiece su andadura por debajo de la superficie, su vocación y la del que lo ha escrito no es permanecer en el subsuelo, sino elevarse todo lo posible, incluso mucho más allá de lo que la prudencia aconsejaría, aunque sin dejar ni un solo instante de medir los riesgos asumidos.

\section{5. ¿CÓMO PUEDO EVITAR QUE LOS ARISTOTÉLICOS SE ENFADEN CONMIGO?}

Como advertí al comienzo, una de las pocas escuelas de pensamiento que no ha renegado de la filosofía de la naturaleza es la aristotélica. Sin embargo, 
a los miembros de esa corriente mi aristotelismo parecerá bastante dudoso, cuando no inexistente o destestable. Me apresuro a confesar que no soy especialista en Aristóteles ni lo pretendo, aunque sostengo que nadie tiene el monopolio de los problemas ni de los pensadores, por mucho que debamos respetar la autoridad de los que han consagrado años de esfuerzos a un autor o una problemática.

He leído bastantes escritos del filósofo griego y después de hacerlo saqué en limpio que en lo tocante al saber era un posibilista y que para él los conocimientos que proporciona la razón están orgánicamente entrelazados. Algunos especialistas que han sabido conservar la perspectiva de conjunto refrendaron estas convicciones. Uno de ellos me llamó la atención sobre este texto, de los Segundos analíticos: "Es difícil estar seguro de si uno conoce o no, porque es difícil estar seguro de si el conocimiento de uno se basa en las verdades fundamentales apropiadas a cada atributo, que es la diferencia característica del verdadero conocimiento”. En mi opinión, avala la tesis capital de mi libro: en la verdad, y no en la evidencia ni en la certeza, radica el valor de una empresa cognitiva.

Evidencias y certezas no dejan de ser concomitancias objetivas y subjetivas de la verdad; buenas y deseables cuando están disponibles, pero en modo alguno imprescindibles. Sobre todo, carecen de legitimidad para hipotecar la verdad, y en ningún caso se pueden erigir en conditio sine qua non de ella. Es el primer punto en que me reivindico como aristotélico.

El segundo tiene que ver con el hecho indiscutible de que la separación de la física con respecto a la metafísica fue llevada a cabo por bibliotecarios y no por el propio Aristóteles. Hay disquisiciones teológicas en los libros de Física 
Naturaleza y Libertad. Revista de estudios interdisciplinares. Número 3, 2014. ISSN: 2254-9668

y referencias bien concretas y empíricas en los de Metafisica. También en esto pretendo seguirle.

Desde que Galileo y compañía echaron abajo la física de su maestro, los aristotélicos empezaron a reivindicar que física y metafísica son dos vagones fácilmente desenganchables. Hoy día casi todos repiten que mientras su cosmología está completamente superada, en filosofía primera sigue siendo insuperable. Personalmente pienso que ni su física era tan mala ni su metafísica tan perenne y monolítica. En cambio me parece una desgracia que dejaran de ir de la mano. El conocimiento empírico ha crecido muchísimo en los últimos dos mil años. Una buena metafísica aristotélica tendría en este sentido que comenzar por una reconstrucción de la física. Mi libro quisiera ser situado en los prolegómenos de esa ilusionante tarea.

Juan Arana

jarana@us.es 